\title{
Atomic order and metallic nanoclusters in $\mathrm{Na}_{4} \mathrm{Ir}_{3} \mathrm{O}_{8}$
}

\author{
M. V. Talanov ${ }^{1, *}$, V. B. Shirokov ${ }^{2}$, V. M. Talanov ${ }^{3}$ \\ ${ }^{1}$ Research Institute of Physics, Southern Federal University, Rostov-on-Don, Russia \\ ${ }^{2}$ Southern Scientific Center of Russian Academy of Sciences, Rostov-on-Don, Russia \\ ${ }^{3}$ South-Russian State Polytechnical University, Novocherkassk, Russia \\ tmikle-man@mail.ru,shirokov-vb@rambler.ru, *valtalanov@mail.ru
}

PACS 61.50.Ks; 36.40.-c

DOI 10.17586/2220-8054-2015-6-3-442-450

\begin{abstract}
A theory for forming Ir-atomic nanoclusters in a dielectric matrix of $\mathrm{Na}_{4} \mathrm{Ir}_{3} \mathrm{O}_{8}$ structure and spinel-like structures is suggested. The atomic order in the $\mathrm{Na}_{4} \mathrm{Ir}_{3} \mathrm{O}_{8}$ structure is investigated by group-theoretical methods of phase transition theory. The critical irreducible representation $\tau$, generating appearance of enantiomorphic $P 4_{1} 32\left(P 4_{3} 32\right)$-phases from high symmetry spinel-like phase with space group Fd3m, is six dimensional irreducible representation $\mathrm{k}_{10}\left(\tau_{1}\right)$ (in Kovalev designation). Ir and $\mathrm{Na}$ atoms form an intriguing atom ordering, giving rise to a network of corner shared Ir triangles, called a hyperkagome lattice. It is shown that inside the hyperkagome lattice, there are closed metal contours of chemical bonds formed by Ir-clusters - decagons. Unusual physical properties of solid solutions on the basis of $\mathrm{Na}_{4} \mathrm{Ir}_{3} \mathrm{O}_{8}$ are expected. The existence of hyperkagome lattices in six types in ordered spinel structures is theoretically predicted.
\end{abstract}

Keywords: hyper-kagome order, ordered spinels, decagons, nanoclusters.

Received: 7 January 2015

Revised: 24 February 2015

\section{Introduction}

Oxides with the $\mathrm{A}\left[\mathrm{B}_{2}\right] \mathrm{O}_{4}$ spinel structure containing atoms of transition elements in octahedral positions (atoms B), are characterized by a large variety of physical properties, among which are superconductivity, charging ordering, heavy fermion behavior, unusual magnetic properties in strong magnetic fields and others. In many cases, these important physical properties are connected with a structural feature of spinels - a three-dimensional network of the tetrahedra formed by octahedral B-cations. A network of these tetrahedra is called a pyrochlore lattice, for B-cations are formed in pyrochlore structure such a sublattice as B-cations in spinel structure. P.W. Anderson was the first who showed that B- sublattice in spinel structures was geometrically frustrated and caused unusual physical properties of this class of materials [1]. The term "geometrical frustration" describes structures with local order generated by lattice geometry. Frustration arises when the geometry of a system allows for a set of degenerate ground states. Such highly degenerate systems are extremely sensitive to thermal and quantum fluctuations, and thereby intriguing classical and quantum ground states may emerge via "order by disorder".

One of compounds in which it is possible to expect the manifestation of unusual physical properties is $\mathrm{Na}_{4} \mathrm{Ir}_{3} \mathrm{O}_{8}$, which has spinel-like structure. The geometrical frustration of spins in this substance causes the existence of exotic electronic and structural state - spin liquid. The crystals of $\mathrm{Na}_{4} \mathrm{Ir}_{3} \mathrm{O}_{8}$ are the only substances in which an electronic subsystem is $3 \mathrm{D}$ spin liquid [2]. 
The purpose of this work is the theoretical research of the symmetry and structural conditions for the appearance of atomic hyperkagome order in structures of ordered low symmetric modifications of spinels and spinel-like substances.

\section{Atomic hyperkagome order in $\mathrm{Na}_{4} \mathrm{Ir}_{3} \mathrm{O}_{8}$ structures}

The atomic order in $\mathrm{Na}_{4} \mathrm{Ir}_{3} \mathrm{O}_{8}$ was investigated by group-theoretical methods of phase transition theory. Structural studies allowed the authors [2] to establish space group $\mathrm{P} 4_{1} 32$ or $\mathrm{P}_{3} 32$ and some features of the $\mathrm{Na}_{4} \mathrm{Ir}_{3} \mathrm{O}_{8}$ structure. It was shown that in this structure there is hyperkagome sublattice in positions of which there are $\operatorname{Ir}^{4+}$ ions in a low-spin state $\left(\mathrm{t}_{2 g}^{5}\right)$ with $\mathrm{S}=1 / 2$.

In this paper, we theoretically describe the formation of atom hyperkagome lattice in $\mathrm{Na}_{4} \mathrm{Ir}_{3} \mathrm{O}_{8}$ on the basis of hypothetical parent phase conception. This parent phase has a spinel-like structure (space group $\mathrm{Fd} 3 \mathrm{~m}$ ) and a $\left[\mathrm{Na}_{1 / 2} \mathrm{Ir}_{3 / 2}\right]^{16(d)}\left[\mathrm{Na}_{3 / 2}\right]^{16(\mathrm{c})} \mathrm{O}_{4}^{32(e)}$ composition.

This structure differs from spinel one by the following:

- positions 8 (a) are vacant;

- positions 16 (c), occupied by $75 \% \mathrm{Na}^{1+}$ ions in $\mathrm{Na}_{4} \mathrm{Ir}_{3} \mathrm{O}_{8}$ structure $[2,3]$, are vacant in spinel structure.

The critical order parameter which induces hypothetical phase transition has been stated. The critical irreducible representation (IR) $\tau$, generating appearance of enantiomorphic $P 4_{1} 32\left(P 4_{3} 32\right)$-phases from high symmetry spinel-like phase with space group Fd3m, is six dimensional irreducible representation $\mathbf{k}_{10}\left(\tau_{1}\right)$ (table 1 ). IR designations are given according to Kovalev [4]: $\mathbf{k}_{10}\left(\tau_{i}\right)$ - is the star of wave vector; $\tau_{i}$,- is a number of corresponding IR.

Stratification of Wyckoff positions in spinel-like structure with space group Fd3m occurs as the result of a hypothetical phase transformation that takes place.

The 16(d) position in the parent structure was stratified into positions $4(\mathrm{~b})$ and $12(\mathrm{~d})$ in $P 4_{1} 32\left(P 4_{3} 32\right)$-structure, $16(\mathrm{c})$ position was stratified into positions $4(\mathrm{a})$ and $12(\mathrm{~d})$, the $32(\mathrm{e})$ position was stratified into positions $8(\mathrm{c})$ and 24(e). Taking into account the occupation of 16(c) positions equal to $75 \%$ [2, 3], structural formula $\mathrm{Na}_{4} \mathrm{Ir}_{3} \mathrm{O}_{8}$ in $P 4_{1} 32\left(P 4_{3} 32\right)$-phase can be presented as $\left[\mathrm{Na}_{1 / 2}^{4(b)} \mathrm{Ir}_{3 / 2}^{12(d)}\right]\left[\mathrm{Na}_{3 / 8}^{4(a)} \mathrm{Na}_{9 / 8}^{12(d)}\right] \mathrm{O}^{8(c)} \mathrm{O}_{3}^{24(e)}$.

It should be noted that in $\mathrm{Na}_{4} \mathrm{Ir}_{3} \mathrm{O}_{8}$, there cannot be charge ordering of iridium atoms in principle, for this atom takes only one position $12(\mathrm{~d})$ with site symmetry $2\left(\mathrm{C}_{2}\right)$ in $P 4_{1} 32$ $\left(\mathrm{P}_{3} 32\right)$-phase. This means that all the Ir sites and Ir-Ir bonds are equivalent.

The analysis of mechanical, permutation and orbital representations of parent structure allowed us to establish that that the structure of $\mathrm{Na}_{4} \mathrm{Ir}_{3} \mathrm{O}_{8}$ is formed as a result of atoms displacements of sodium, iridium and oxygen, and also atoms ordering of sodium, iridium and oxygen [5-8]. Ordering of all atoms occurs according to the type 1:3. (Fig. 1). Enantiomorphic forms of $\mathrm{Na}_{4} \mathrm{Ir}_{3} \mathrm{O}_{8}$ are mirror antipodes. Their structures coincide with each other only in the reflection in the mirror (Fig. 1). Chemical and physical properties of the enantiomorphic modifications of $\mathrm{Na}_{4} \mathrm{Ir}_{3} \mathrm{O}_{8}$ are the same (except optical activity) [9-15].

In the structure of this substance there is a pyrochlore sublattice in which each tetrahedron is occupied by three $\mathrm{Ir}$ and one $\mathrm{Na}^{12(d)}$. These Ir and $\mathrm{Na}$ atoms form an intriguing atom ordering, giving rise to a network of corner shared Ir triangles, called a hyperkagome lattice [14].

Metal atoms in $\mathrm{Na}_{4} \mathrm{Ir}_{3} \mathrm{O}_{8}$ structure form two different networks of tetrahedra: $\left[\mathrm{Na}_{3}^{12(d)}\right.$ $\left.\mathrm{Na}^{4(a)}\right]$ and $\left[\operatorname{Ir}_{3}^{12(d)} \mathrm{Na}^{4(b)}\right]$ (Fig. 2). Each of these networks forms its own hyperkagome sublattice. The hyperkagome sublattice in the network of $\left[\mathrm{Na}_{3}^{12(d)} \mathrm{Na}^{4(a)}\right]$ tetrahedra is formed 
TABLE 1. Symmetrically structural identification of the phase states in lowsymmetry phases $\left(\mathrm{G}_{D}\right)$ induced by the IR $\mathrm{k}_{10}\left(\tau_{1}\right)$ of the space group $\mathrm{Fd} 3 \mathrm{~m}$

\begin{tabular}{|c|c|c|c|c|}
\hline \multirow{2}{*}{$\vec{\varphi}$} & \multirow{2}{*}{$G_{D}$} & \multicolumn{3}{|c|}{ Splitting of Wyckoff position of spinel-like structure of parent phase } \\
\hline & & $8(\mathrm{a})$ & $16(\mathrm{c}), 16(\mathrm{~d})$ & $32(\mathrm{e})$ \\
\hline $0 \varphi 0 \varphi 0-\varphi$ & $\begin{array}{l}\mathrm{P} 4_{1} 32 \\
\left(\mathrm{P} 4_{3} 32\right)\end{array}$ & $1(8): 3\left(\mathrm{C}_{3}\right)$ & $1(4): 32\left(\mathrm{D}_{3}\right)+1(12): 2\left(\mathrm{C}_{2}\right)$ & $1(8): 3\left(\mathrm{C}_{3}\right)+1(24): 1\left(\mathrm{C}_{1}\right)$ \\
\hline$\varphi \varphi \varphi \varphi \varphi \varphi$ & $\mathrm{R} \overline{3} \mathrm{~m}$ & $\begin{array}{l}1(4): 3 \mathrm{~m}\left(\mathrm{C}_{3 v}\right)+ \\
+1(12): \mathrm{m}\left(\mathrm{C}_{s}\right) \\
\end{array}$ & $\begin{array}{l}1(2): \overline{3} \mathrm{~m}\left(\mathrm{D}_{3 d}\right)+1(6): 2 / \mathrm{m}\left(\mathrm{C}_{2 h}\right)+ \\
\quad+1(12): \mathrm{m}\left(\mathrm{C}_{s}\right)+1(12): 2\left(\mathrm{C}_{2}\right)\end{array}$ & $\begin{array}{l}1(4): 3 \mathrm{~m}\left(\mathrm{C}_{3 v}\right)+3(12): \\
: \mathrm{m}\left(\mathrm{C}_{s}\right)+1(24): 1\left(\mathrm{C}_{1}\right)\end{array}$ \\
\hline $00000 \varphi$ & $\begin{array}{c}\mathrm{P} 4_{1} 22 \\
\left(\mathrm{P} 4_{3} 22\right) \\
\end{array}$ & $1(16): 2\left(\mathrm{C}_{2}\right)$ & $2(16): 2\left(\mathrm{C}_{2}\right)$ & $2(32): 1\left(\mathrm{C}_{1}\right)$ \\
\hline $0 \varphi 00 \varphi 0$ & $\mathrm{P} \overline{4} 2_{1} \mathrm{~m}$ & $\begin{array}{c}1(4): \mathrm{mm} 2\left(\mathrm{C}_{2 v}\right)+1(4): \\
: \overline{4}\left(\mathrm{~S}_{4}\right)+1(8): \mathrm{m}\left(\mathrm{C}_{s}\right)\end{array}$ & $2(8): \mathrm{m}\left(\mathrm{C}_{s}\right)+1(16): 1\left(\mathrm{C}_{1}\right)$ & $4(8): \mathrm{m}\left(\mathrm{C}_{s}\right)+2(16): 1\left(\mathrm{C}_{1}\right)$ \\
\hline $00 \varphi \varphi 00$ & $\mathrm{Pbmn}$ & $1(16): \mathrm{m}\left(\mathrm{C}_{s}\right)$ & $2(8): 2 / \mathrm{m}\left(\mathrm{C}_{2 h}\right)+1(16): 2\left(\mathrm{C}_{2}\right)$ & $2(16): \mathrm{m}\left(\mathrm{C}_{s}\right)+1(32): 1\left(\mathrm{C}_{1}\right)$ \\
\hline$\varphi_{1} \varphi_{2} \varphi_{1} \varphi_{2} \varphi_{1} \varphi_{2}$ & R32 & $\begin{array}{l}1(4): 3\left(\mathrm{C}_{3}\right)+ \\
+1(12): 1\left(\mathrm{C}_{1}\right)\end{array}$ & $\begin{array}{c}1(2): 32\left(\mathrm{D}_{3}\right)+3(6): 2\left(\mathrm{C}_{2}\right)+ \\
+1(12): 1\left(\mathrm{C}_{1}\right)\end{array}$ & $1(4): 3\left(\mathrm{C}_{3}\right)+5(12): 1\left(\mathrm{C}_{1}\right)$ \\
\hline$\varphi_{1} 00 \varphi_{2} 00$ & $\mathrm{P} 2{ }_{1} 2_{1} 2$ & $\begin{array}{l}2(4): 2\left(\mathrm{C}_{2}\right)+ \\
+1(8): 1\left(\mathrm{C}_{1}\right)\end{array}$ & $4(8): 1\left(\mathrm{C}_{1}\right)$ & $8(8): 1\left(C_{1}\right)$ \\
\hline $0000 \varphi_{1} \varphi_{2}$ & $\mathrm{P} 222_{1}$ & $1(16): 1\left(\mathrm{C}_{1}\right)$ & $4(8): 2\left(\mathrm{C}_{2}\right)$ & $4(16): 1\left(\mathrm{C}_{1}\right)$ \\
\hline $0 \varphi_{1} 0 \varphi_{2} 0-\varphi_{1}$ & $\begin{array}{r}\mathrm{P} 4_{1} 2_{1} 2 \\
\left(\mathrm{P} 4_{3} 2_{1} 2\right) \\
\end{array}$ & $1(16): 1\left(\mathrm{C}_{1}\right)$ & $2(8): 2\left(\mathrm{C}_{2}\right)+1(16): 1\left(\mathrm{C}_{1}\right)$ & $4(16): 1\left(\mathrm{C}_{1}\right)$ \\
\hline$\varphi_{1} \varphi_{1} \varphi_{2} \varphi_{2}-\varphi_{1}-\varphi_{1}$ & $\mathrm{C} 2 / \mathrm{m}$ & $\begin{array}{l}2(4): \mathrm{m}\left(\mathrm{C}_{s}\right)+ \\
+1(8): 1\left(\mathrm{C}_{1}\right)\end{array}$ & $\begin{array}{c}2(2): 2 / \mathrm{m}\left(\mathrm{C}_{2 h}\right)+1(4): \overline{1}\left(\mathrm{C}_{i}\right)+ \\
+1(4): \mathrm{m}\left(\mathrm{C}_{s}\right)+1(4): 2\left(\mathrm{C}_{2}\right)+ \\
+2(8): 1\left(\mathrm{C}_{1}\right)\end{array}$ & $4(4): \mathrm{m}\left(\mathrm{C}_{s}\right)+6(8): 1\left(\mathrm{C}_{1}\right)$ \\
\hline$\varphi_{1} 0 \varphi_{2} 0 \varphi_{3} 0$ & $\mathrm{P} 2{ }_{1} 2_{1} 2_{1}$ & $2(8): 1\left(\mathrm{C}_{1}\right)$ & $4(8): 1\left(\mathrm{C}_{1}\right)$ & $8(8): 1\left(C_{1}\right)$ \\
\hline$\varphi_{1} 0 \varphi_{2} \varphi_{3}-\varphi_{1} 0$ & $\mathrm{C} 222_{1}$ & $2(8): 1\left(\mathrm{C}_{1}\right)$ & $4(4): 2\left(\mathrm{C}_{2}\right)+2(8): 1\left(\mathrm{C}_{1}\right)$ & $8(8): 1\left(\mathrm{C}_{1}\right)$ \\
\hline$\varphi_{1} \varphi_{1} \varphi_{2} \varphi_{2} \varphi_{3} \varphi_{3}$ & $\mathrm{P} \overline{1}$ & $4(4): 1\left(\mathrm{C}_{1}\right)$ & $\mathrm{v} 4(2): \overline{1}\left(\mathrm{C}_{i}\right)+6(4): 1\left(\mathrm{C}_{1}\right)$ & $16(4): 1\left(\mathrm{C}_{1}\right)$ \\
\hline$\varphi_{1} \varphi_{1} \varphi_{2} \varphi_{3} \varphi_{3} \varphi_{2}$ & $\mathrm{Cm}$ & $\begin{array}{l}4(2): \mathrm{m}\left(\mathrm{C}_{s}\right)+ \\
+2(4): 1\left(\mathrm{C}_{1}\right)\end{array}$ & $4(2): \mathrm{m}\left(\mathrm{C}_{s}\right)+6(4): 1\left(\mathrm{C}_{1}\right)$ & $8(2): \mathrm{m}\left(\mathrm{C}_{s}\right)+12(4): 1\left(\mathrm{C}_{1}\right)$ \\
\hline$\varphi_{1} \varphi_{2} \varphi_{3} \varphi_{4} \varphi_{3} \varphi_{4}$ & $\mathrm{C} 2$ & $4(4): 1\left(C_{1}\right)$ & $4(2): 2\left(\mathrm{C}_{2}\right)+6(4): 1\left(\mathrm{C}_{1}\right)$ & $16(4): 1\left(C_{1}\right)$ \\
\hline $0 \varphi_{1} 0 \varphi_{2} \varphi_{3} \varphi_{4}$ & $\mathrm{P} 2_{1}$ & $4(4): 1\left(C_{1}\right)$ & $8(4): 1\left(\mathrm{C}_{1}\right)$ & $16(4): 1\left(\mathrm{C}_{1}\right)$ \\
\hline$\varphi_{1} \varphi_{2} \varphi_{3} \varphi_{4} \varphi_{5} \varphi_{6}$ & $\mathrm{P} 1$ & $8(2): 1\left(C_{1}\right)$ & $16(2): 1\left(\mathrm{C}_{1}\right)$ & $32(2): 1\left(\mathrm{C}_{1}\right)$ \\
\hline
\end{tabular}

Notes. Designation for order parameters $\mathrm{k}_{10}-\varphi$. One of the enantiomorphic space groups is recorded in parentheses in the second column. The number and multiplicity (in parentheses) of the Wyckoff position are indicated. After the colon, the local symmetry of the Wyckoff position is given. For example, the record of $1(8): 3\left(\mathrm{C}_{3}\right)+1(24): 1\left(\mathrm{C}_{1}\right)$ means that Wyckoff position 32e of group $\mathrm{Fd} 3 \mathrm{~m}$ in the low-symmetry phase $\mathrm{G}_{D}$ with space group $\mathrm{P}_{1} 32$ stratified into one eightfold Wyckoff position with local symmetry $3\left(\mathrm{C}_{3}\right)$ and one 24 -fold Wyckoff position with local symmetry $1\left(\mathrm{C}_{1}\right)$. 

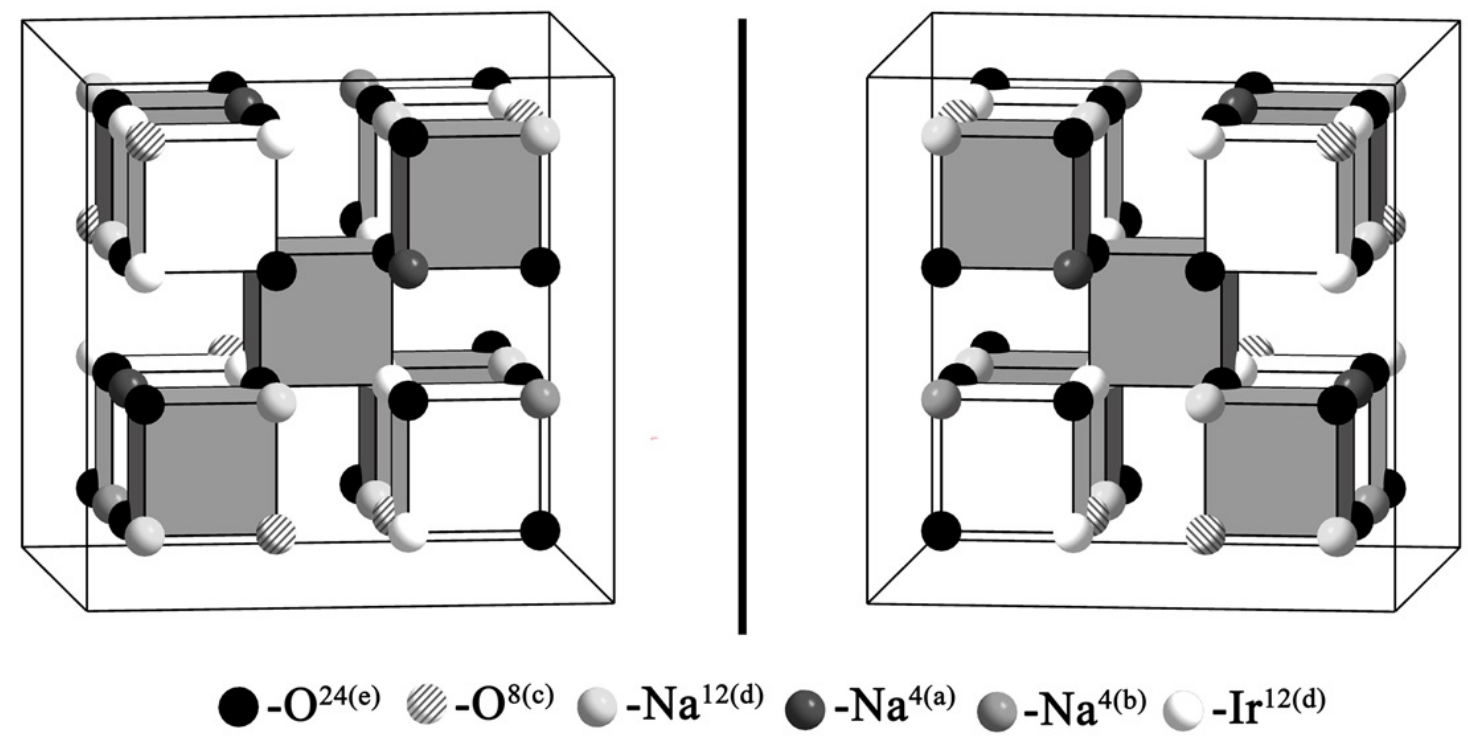

FIG. 1. Ordered cation and anion distributions in enantiomorphic structures $\mathrm{P}_{3} 32$ - and $\mathrm{P} 4{ }_{1} 31-\mathrm{Na}_{4} \mathrm{Ir}_{3} \mathrm{O}_{8}$ modifications (without atoms displacements). The line between the structures means mirror

by $\left[\mathrm{Na}_{3}^{12(d)}\right]_{n}$ triangles. This hyperkagome sublattice has an artificial character from the point of view of physics because there is no principal difference in properties of $\mathrm{Na}^{12(d)}$ and $\mathrm{Na}^{4(a)}$ ions. The $\left[\mathrm{Ir}_{3}^{12(d)}\right]_{n}$ - hyperkagome sublattice in $\mathrm{Na}_{4} \mathrm{Ir}_{3} \mathrm{O}_{8}$ structure has significant physical importance. In this hyperkagome sublattice, in each tetrahedron, three vertices occupy magnetic iridium ions $\operatorname{Ir}^{12(d)}$ and one vertex is occupied by a sodium ion $\mathrm{Na}^{4(b)}$.

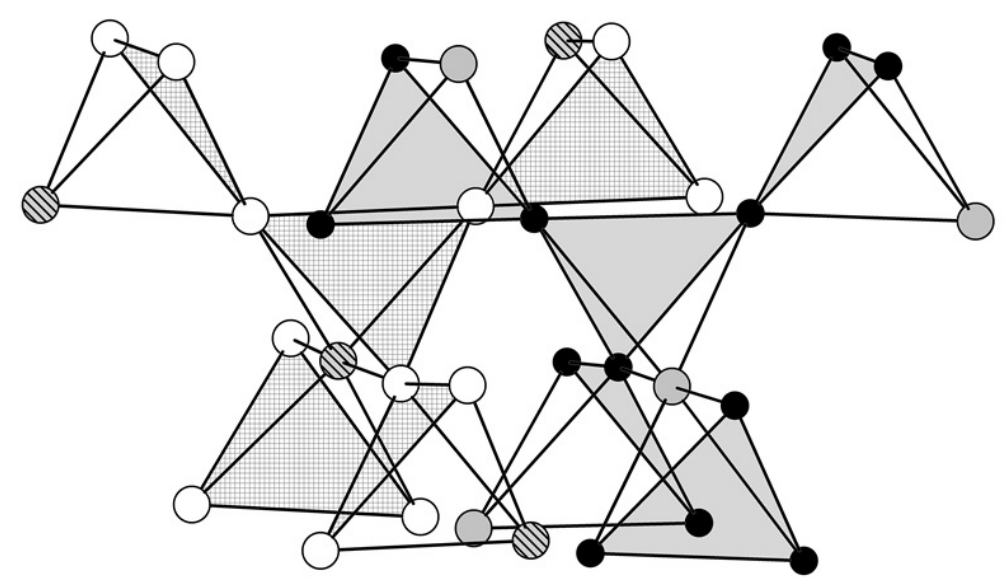

FIG. 2. Two networks of tetrahedra $\left[\mathrm{Na}_{3}^{12(d)} \mathrm{Na}^{4(a)}\right]$ and $\left[\operatorname{Ir}_{3}^{12(d)} \mathrm{Na}^{4(b)}\right]$ forming two hyper-kagome sublattices. One sublattice atoms formed Ir atoms (this sublattice is painted in gray), the second sublattice formed by atoms $\mathrm{Na}^{12(d)}$ (this sublattice is hatched pattern)

Four tetrahedra form hypertetrahedra $\left[\operatorname{Ir}_{3}^{12(d)} \mathrm{Na}^{4(b)}\right]_{4}$ (Fig. 3). A hyperkagome-lattice is formed from a network of corner shared $\left[\operatorname{Ir}_{3}^{12(d)} \mathrm{Na}^{4(b)}\right]_{4}$ tetrahedra as follows: in every tetrahedron a right triangle $\left[\operatorname{Ir}_{3}^{12(d)}\right]$ is formed from iridium atoms. 


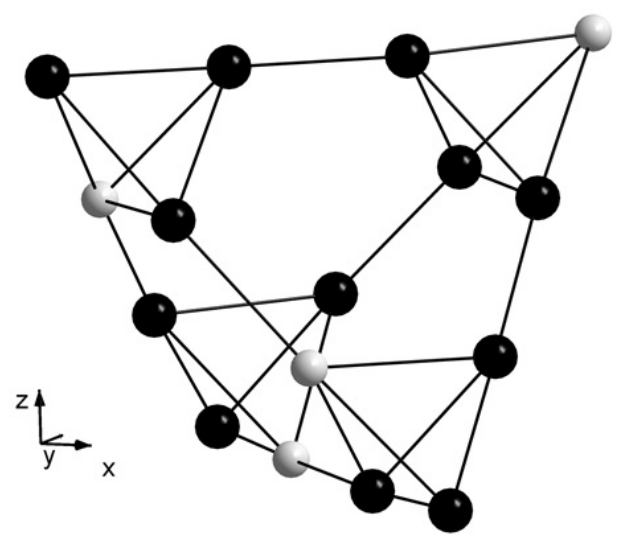

FIG. 3. Hypertetrahedron $\left[\operatorname{Ir}_{3}^{12(d)} \mathrm{Na}^{4(b)}\right]_{4}$ in structure $\mathrm{Na}_{4} \mathrm{Ir}_{3} \mathrm{O}_{8}$

As all Ir positions in structure of $\mathrm{Na}_{4} \mathrm{Ir}_{3} \mathrm{O}_{8}$ are equivalent, all distances of Ir-Ir are identical and equal to $3.112 \AA$. These right triangles form a network $\left[\operatorname{Ir}_{3}^{12(d)}\right]_{n}$. (Fig. 4). This network of connected triangles forms unique atom hyperkagome order [2] (Fig. 5, 6).

In a hyperkagome lattice, it is possible to allocate the module from three crossed minimum closed contours (Fig.7). These contours are formed by Ir-Ir chemical bonds. The module consists of 14 iridium triangles. Two of these triangles are general for all three minimum contours. The minimum (or shortest) closed contour includes ten Ir-Ir bonds. These ten Ir atoms form a crown-like cluster - so-called decagon [8] (Fig. 8). The perimeter of all minimum contours is identical and equal to $31.12 \AA$. The greatest distance between opposite Ir atoms in decagon is equal to $10.775 \AA$, and its maximum width $-5.659 \AA$.

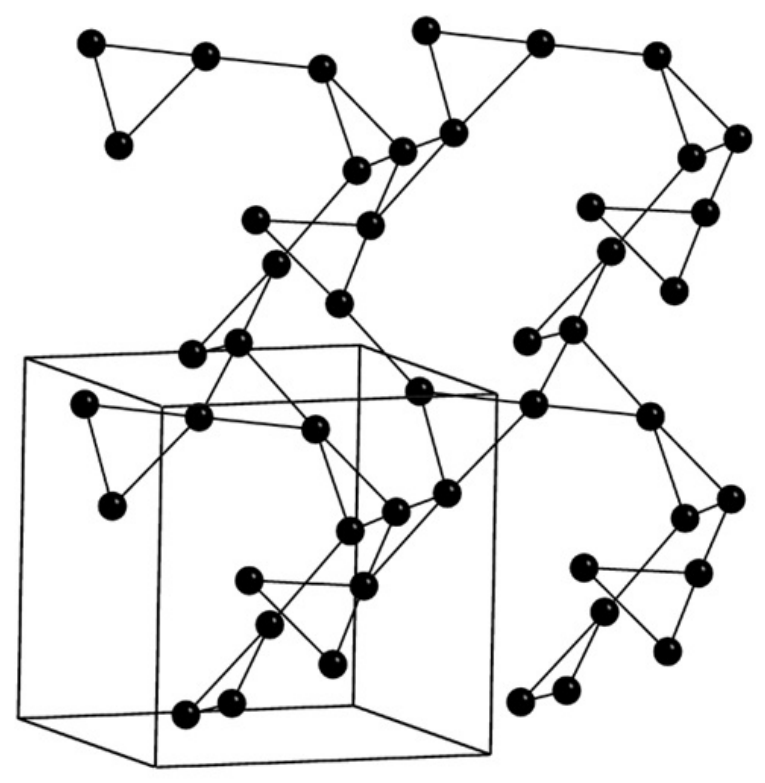

Fig. 4. A hyperkagome sublattice. A hyperkagome sublattice is a cubic lattice with 12 sites in a unit cell, it is a network of corner-sharing Ir-triangles

$\mathrm{P}_{1} 32\left(\mathrm{P} 4_{3} 32\right)$-spinel phases are widely spread types of low-symmetry ordered enantiomorphic modifications of spinel structures. They are formed as a result of phase transitions from spinel structures. These phases are generated by critical irrep $\mathbf{k}_{10}\left(\tau_{1}\right)[5,6,14]$. Irrep 


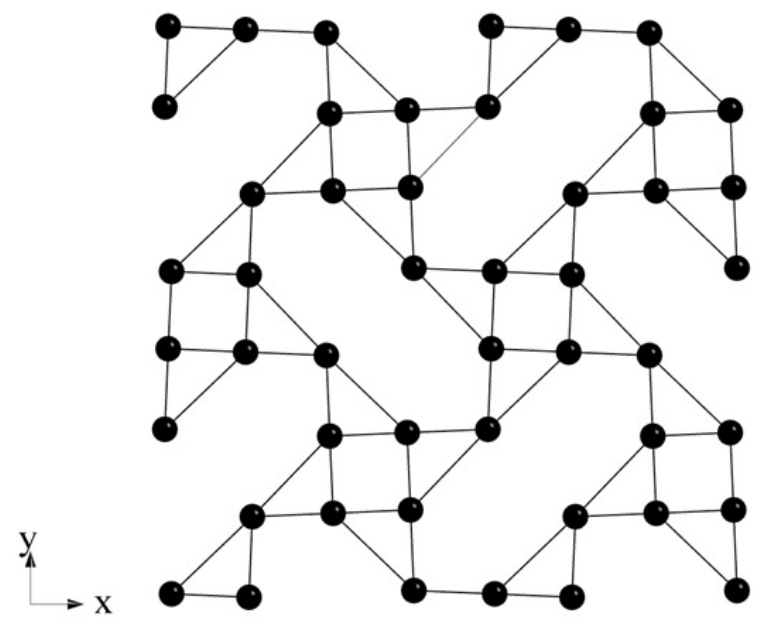

FIG. 5. A hyperkagome sublattice. View of hyperkagome sublattice along the [001] axes

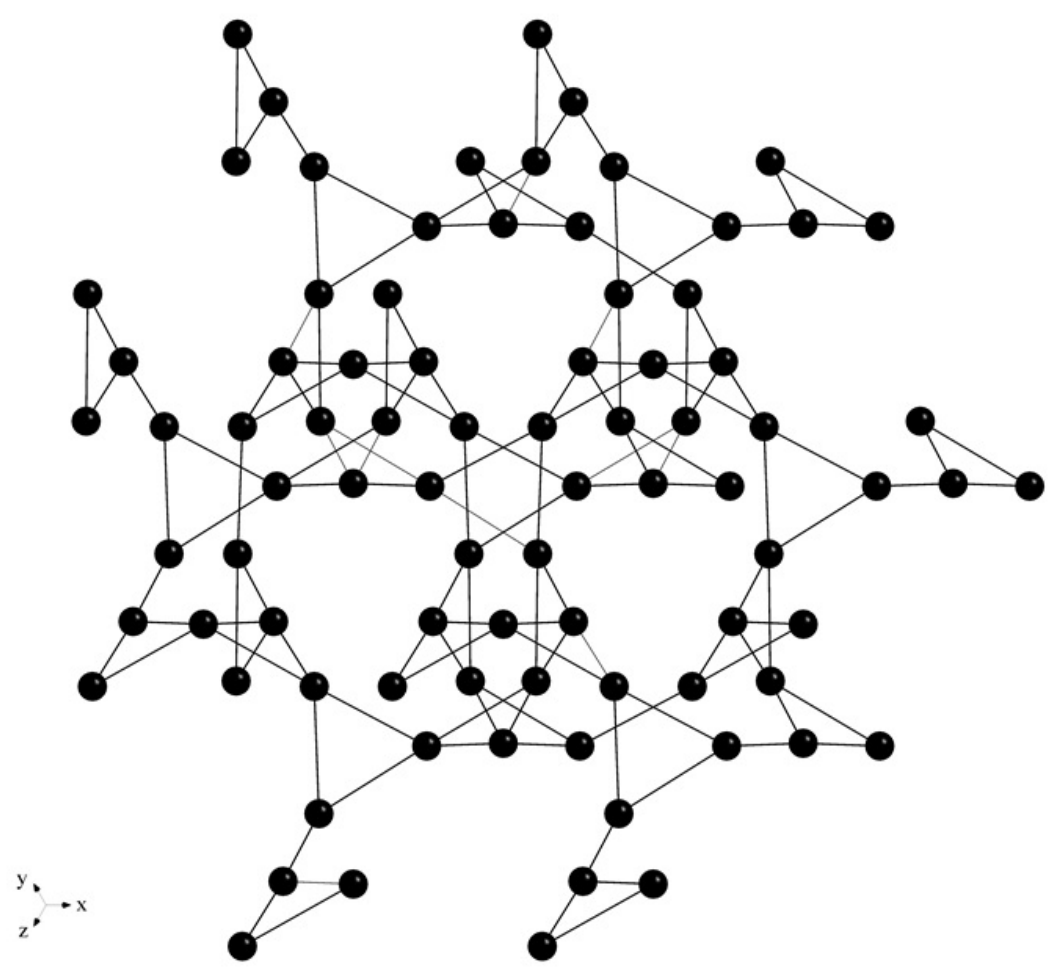

FIG. 6. A hyperkagome sublattice: general picture 
TABLE 2. The types of binary cation ordering of 1:3 types in Wyckoff position 16(d) of spinel structure

\begin{tabular}{|c|c|c|c|c|}
\hline $\mathrm{N}$ & Order parameters & $\begin{array}{l}\text { Symbol of } \\
\text { space group }\end{array}$ & $\begin{array}{l}\mathrm{V}_{0} \\
/ \mathrm{V}\end{array}$ & Structural formula \\
\hline 1 & $(\xi,-\xi, \xi)^{7}$ & $\mathrm{R} \overline{3} 2 / \mathrm{m}(\mathrm{N} 166)$ & 1 & $\mathrm{~A}^{(c)} \mathrm{B}_{1 / 2}^{(b)} \mathrm{B}_{3 / 2}^{(e)} \mathrm{X}^{(c)} \mathrm{X}_{3}^{(h)}$ \\
\hline 2 & $(\varphi, 0, \varphi, 0, \varphi, 0)^{3}$ & $\mathrm{P} \overline{4} 3 \mathrm{~m}(\mathrm{~N} 215)$ & 4 & $\mathrm{~A}_{1 / 8}^{(a)} \mathrm{A}_{3 / 8}^{(c)} \mathrm{A}_{1 / 2}^{(e)} \overline{\mathrm{B}_{1 / 2}^{(e)} \mathrm{B}_{3 / 2}^{(i)}} \mathrm{X}_{1 / 2}^{(e)} \mathrm{X}_{1 / 2}^{(e)} \mathrm{X}_{3 / 2}^{(i)} \mathrm{X}_{3 / 2}^{(i)}$ \\
\hline 3 & $(0, \varphi, 0, \varphi, 0,-\varphi)^{1}$ & $\begin{array}{l}\mathrm{P} 4_{3} 32(\mathrm{~N} 212) \\
\mathrm{P} 4_{1} 32(\mathrm{~N} 213)\end{array}$ & 4 & $\mathrm{~A}^{(c)} \overline{\mathrm{B}_{1 / 2}^{(a)} \mathrm{B}_{3 / 2}^{(d)}} \mathrm{X}^{(c)} \mathrm{X}_{3}^{(e)}$ \\
\hline 4 & $(0, \varphi, 0, \varphi, 0,-\varphi)^{3}(\xi)^{4}$ sec. & $\mathrm{P} \overline{4} 3 \mathrm{~m}(\mathrm{~N} 215)$ & 4 & $\mathrm{~A}_{1 / 8}^{(a)} \mathrm{A}_{3 / 8}^{(c)} \mathrm{A}_{1 / 2}^{(e)} \mathrm{B}_{1 / 2}^{(e)} \mathrm{B}_{3 / 2}^{(i)} \mathrm{X}_{1 / 2}^{(e)} \mathrm{X}_{1 / 2}^{(e)} \mathrm{X}_{3 / 2}^{(i)} \mathrm{X}_{3 / 2}^{(i)}$ \\
\hline 5 & $(\xi)^{4, A}(\xi,-\xi, \xi)^{7}$ & R3m (N160) & 1 & 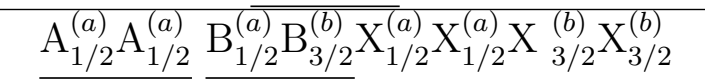 \\
\hline 6 & $(\xi)^{4}(0, \varphi, 0, \varphi, 0,-\varphi)^{1}$ & $\mathrm{P} 2_{1} 3$ (N198) & 4 & $\mathrm{~A}_{1 / 2}^{(a)} \mathrm{A}_{1 / 2}^{(a)} \overline{\mathrm{B}_{1 / 2}^{(a)} \mathrm{B}_{3 / 2}^{(b)}} \mathrm{X}_{1 / 2}^{(a)} \mathrm{X}_{1 / 2}^{(a)} \mathrm{X}_{3 / 2}^{(b)} \mathrm{X}_{3 / 2}^{(b)}$ \\
\hline
\end{tabular}

$\mathbf{k}_{10}\left(\tau_{1}\right)$ enters into both permutation (on Wyckoff position $16(\mathrm{~d})$ and $32(\mathrm{e})$ ) and mechanical (on Wyckoff position 8(a), 16(d), 32(e)) representations of spinel structure. Therefore, the lowering of crystal symmetry is due to displacements of all atom types and ordering of octahedral cations and anions (in both cases the type of order is 1:3). Calculated results show that atoms $\mathrm{A}^{8(c)}$ are located in weakly distorted tetrahedra, atoms $\mathrm{B}^{612(d)}$ are in distorted octahedra, atoms $\mathrm{B}^{4(b)}$ are present in regular octahedra (Fig. $11 \mathrm{a}$, b). Structure formula of low-symmetry phases has the following view: $\mathrm{A}_{2}^{8(c)} \mathrm{B}^{4(b)} \mathrm{B}_{3}^{(12(d)} \mathrm{X}_{2}^{8(c)} \mathrm{X}_{6}^{24(e)}[5,6]$. The existence of 6 new types of hyperkagome lattice in spinel ordered structures has been theoretically predicted on the basis of group-theoretical analysis results (table 2).

The hyperkagome sublattice in $\mathrm{P}_{1} 32\left(\mathrm{P} 4_{3} 32\right)$-phase structure is formed by triangels $\left[\mathrm{B}_{3}^{{ }^{12(d)}}\right]_{n}$. Each of these sublattices is topologically the same as hyperkagome sublattice in $\mathrm{Na}_{4} \mathrm{Ir}_{3} \mathrm{O}_{8}$ structure. However the surroundings of hyperkagome sublattices in $\mathrm{P} 4_{1} 32\left(\mathrm{P} 4_{3} 32\right)-$ phase structures and in $\mathrm{Na}_{4} \mathrm{Ir}_{3} \mathrm{O}_{8}$ structure are different.

Designations for order parameters: $\mathrm{k}_{10}-\varphi, \mathrm{k}_{11}-\xi$. The superscript index after the closing parenthesis is the representation number according to $[5,6]$ and $\mathrm{V}_{0} / \mathrm{V}$ is the multiplication of primitive cell volume as a result of the structural phase transition. The superscript index in the structural formula means the type of Wyckoff position according to International Tables for Crystallography. In the structural formulas, ordered cations are underlined.

Note that the hyperkagome atomic order was first predicted theoretically in our paper [16], in which aspects of the cation ordering (type 1: 3) in the spinel structure was studied. Atomic order, shown in Fig.1c and 2 [16], is a hyperkagome order. It is exactly the same as in Fig. 4-6 of this work. The name of the hyperkagome order (sublattice) was proposed in $[2]$.

Antiferromagnets with spin $\mathrm{S}=1 / 2$ on kagome- and hyperkagome lattices are rare substances, exotic structure-electronic states of matter. Scientists hope that these materials will find important applications. The theory proposed is in accordance with experimental results [2]. Unusual physical properties are expected to appear when doping $\mathrm{Na}_{4} \mathrm{Ir}_{3} \mathrm{O}_{8}$ crystals. It is known that crystals with broken mirror symmetry show macroscopic effects which were prohibited in centrosymmetric crystals $[17,18]$. The theory predicted three types 


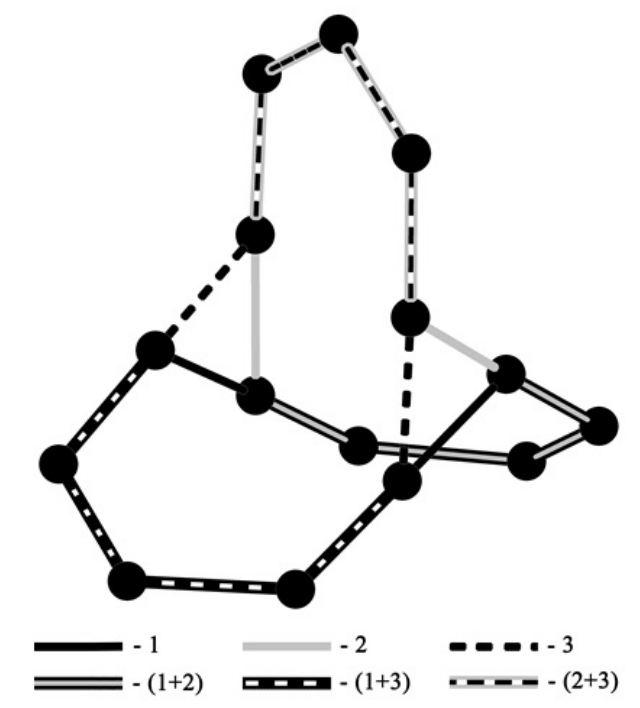

Fig. 7. Structure module of $\mathrm{Na}_{4} \mathrm{Ir}_{3} \mathrm{O}_{8}$, consisting of three minimal intercrossing contours (loops) of Ir-Ir bonds. Each type of contours is designated by one number. If the Ir-Ir bond included in the two contours, they designated as the sum of two numbers

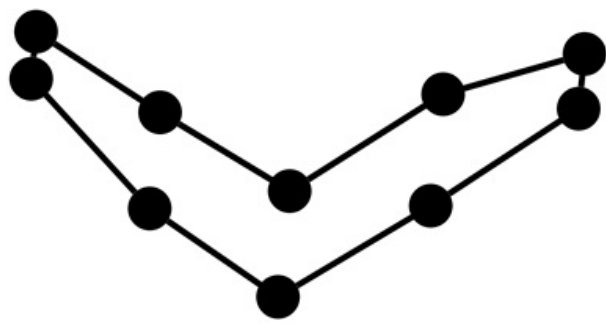

FIG. 8. Nanocluster "decagon" in $\mathrm{Na}_{4} \mathrm{Ir}_{3} \mathrm{O}_{8}$ structure

of structures that can realize a network of corner-sharing tetrahedral (atom hyperkagome order): spinels $\mathrm{AB}_{2} \mathrm{O}_{4}$, pyrochlores $\mathrm{A}_{2} \mathrm{~B}_{2} \mathrm{O}_{7}$ and $\mathrm{C} 15$ Laves phases $\mathrm{AB}_{2}$.

The results of this work have been obtained with the support of the Ministry of Education and Science of the Russian Federation in the framework of the State tasks (Talanov V.M., project N 2983(9.14), (Talanov M.V., Federal program: state contract N 14.575.21.0007; theme Nos. 213.01-11/2014-21, 3.1246.2014/K, 213.01-2014/012-BГ).

\section{References}

[1] Anderson P. Ordering and Antiferromagnetism in Ferrites. Phys. Rev., 1956, 102, P. 1008-1013.

[2] Okamoto Y., Nohara M., Aruga-Katori H., Takagi H. Spin-Liquid State in the S=1/2 Hyperkagome Antiferromagnet $\mathrm{Na}_{4} \mathrm{Ir}_{3} \mathrm{O}_{8}$. Phys. Rev. Let., 2007, 99, P. 137207(4)

[3] Iwasaki M., Takizawa H., Uheda K., Endo T. Synthesis and crystal structure of $\mathrm{Na}_{4} \mathrm{Sn}_{3} \mathrm{O}_{8}$. J. Mater. Chem., 2002, 12, P. 1068-1070.

[4] Kovalev O.V. Representations of Crystallographic Space Groups. Irreducible Representations, Induced Representations and Co-representations London: Taylor and Francis Ltd., 1993, 390 p.

[5] Talanov V.M., Shirokov V.B. Tilting structures in spinels. Acta Crystallographica A, 2012, A68, P. 595606.

[6] Talanov V.M., Shirokov V.B. Atomic order in spinel structure - a group-theoretical analysis. Acta Cryst., 2014, A70, P. 49-63. 
[7] Talanov V.M. Structural mechanism of tetragonal Jahn-Teller distortion of spinels. Izv. Akad. Nauk SSSR. Inorganic materials., 1989, 6, P. 1001-1005.

[8] Talanov V.M. Structural mechanism of the ordering of ions in tetrahedral holes in spinels. Journal of Structural Chemistry, 1986, 2, P. 172-176.

[9] Talanov V.M. Enantiomorphic modifications of spinels. Izv. Akad. Nauk SSSR. Inorganic materials., 1990, 26(11), P. 2428-2430.

[10] Pashchenko N.V., V.M. Talanov. Classification and properties of structural states of crystals. 1. Crystallographic conditions of appearance of extraordinary and irrational structural states Kristallographiya, 1995, 40(6), P. 973-981. Crystallography Reports, 1995, 40(6), P. 900-907.

[11] Pashchenko N.V., V.M. Talanov. Classification and properties of structural states of crystals. 2. Crystallographic conditions of the appearance of isosymmetric, enantiomorphous, and antiisostructural states. Kristallographiya, 1995, 40(6), P. 982-988; Crystallography Reports, 1995, 6, P. 908-914.

[12] Talanov V.M.; Fedorova N.V. Classification and properties of the structural states of crystals: III. Adjacency graphs for the structural states characterized by different numbers of parameters Kristallographiya, 1997, 42(3), P. 394-398; Crystallography Reports, 1997, 3, P. 350-353.

[13] Talanov V.M.; Fedorova N.V. Application of crystallogeometrical theory of structural states of matter to the description of structural transformations in point group 32. Kristallographiya, 1997, 42(3), P. 389-393; Crystallography Reports, 1997, 3, P. 345-349.

[14] Talanov V.M. Theoretical grounds of the natural classification of structure types Kristallographiya, 1996, 44(6), P. 979-997; Crystallography Reports, 1996, 6, P. 929-946.

[15] Ivanov V.V., Talanov V.M. The principle of the modular structure of the nanostructures: information codes and combinatorial design. Nanosystems: physics, chemistry, mathematics, 2010, 1(1), P. 72-107.

[16] Talanov V.M. Structural Modelling of Low-Symmetry Phases of Spinels. II. Phases with 1: 3 Octahedral Order. Phys. Stat. Sol. (b), 1990, 162, P. 339-346.

[17] Ramirez A.P. Handbook on Magnetic Materials. Amsterdam: Elsevier Science, 2001, 13, 423 p.

[18] Bergholtz E.J., Läuchli A. M., Moessner R. Symmetry Breaking on the Three-Dimensional Hyperkagome Lattice of $\mathrm{Na}_{4} \mathrm{Ir}_{3} \mathrm{O}_{8}$. Phys. Rev. Let., 2010, 105, P. 237202(4). 